\title{
USING TWO STACKERS ON ONE RAIL IN A PRODUCTION SYSTEM
}

This paper deals with the possibilities for using two stackers for interoperation transport in a workshop with a technological layout of workplaces. Basic layouts of stacker production systems are discussed. Several strategies for selecting and ordering transport demands in systems with two stackers on one rail have been simulated and evaluated. FIFO strategies are the worst in various layouts of workplaces. On the other hand, the strategy of the choice of the proximate transport demand is simple and very successful. Good technological layout can enhance transport performance.

Keywords: Stacker, material supply, production system, automated transport, optimization, two cooperating stackers.

\section{Introduction}

The rapid progress of electronics, computer science and communication enables economic automation of production processes not only in large series production such as in the automotive industry, but also in piece and low series production, where the technological organization of production does not allow the use of conveyor-belts. The material flow in such production systems is controlled by routings. Transport systems in such production centres should meet two main demands:

- Transfer of material and/or pallets among workplaces and material and tools preparation room;

- Stocking of pallets waiting for operations.

The paper [1] describes an overview of the means of transport.

There are two main automatic transport systems:

- Inductive controlled carts or trains

- Stackers with high racks

The main advantage of inductive carts is that they do not need large structural changes in a workshop, as they do not need rails or barriers for movement of persons in the workshop. The changes in the workshop layout are relatively simple. A breakdown of one cart does not affect production.

The disadvantage of inductive carts is a low inter store capacity.

Stackers unfortunately divide a production center into two halves, and high racks need to be built; but the transport system fulfils not only transport demands but also serves as a middle operation store. Changes in the workshop layout are very difficult, perhaps impossible. The breakdown of a stacker could create big problems in production.

The combination of a stacker storing system and a storage conveyor is mentioned in [2]. The conveyor could be altered by inductive controlled carts or rail guided vehicles.

\section{Basic layouts of stacker production systems}

One stacker, racks and workplaces on both sides

Workplaces in the production centre are situated on both sides of the racks. A stacker works in the aisle between the racks (Fig. 1).

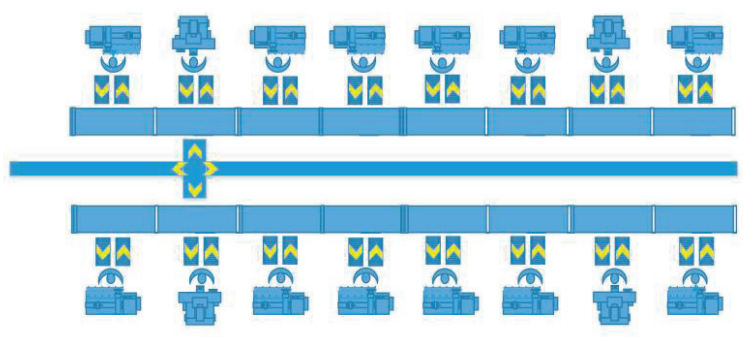

Fig. 1 One stacker, racks and workplaces on both sides

\footnotetext{
* ${ }^{\text {PPavel Kopecek, }}{ }^{1}$ Milan Pinte, ${ }^{2}$ Michal Sasiadek, ${ }^{3}$ Muhammad Latif

${ }^{1}$ University of West Bohemia, Faculty of Mechanical Engineering, Czech Republic

${ }^{2}$ University of Zielona Gora, Poland

${ }^{3}$ School of Engineering, Manchester Metropolitan University, United Kingdom

E-mail: pinte@fst.zcu.cz
} 
If the capacity of the stacker is not sufficient, the production system can be divided into two centres (each with one stacker], or the stacker must be doubled. It is possible to double stackers in three ways:

- Adding one middle rack and using two stackers, each in its own aisle.

- Enlarging the aisle, adding another rail near the former one and using stackers with asymmetric working forks.

- Adding another stacker on the same rail as the former one.

These solutions have many advantages and disadvantages.

Two stackers, racks on both sides with a middle rack, two aisles with one rail and workplaces on both sides of racks

This solution (Fig. 2) is very simple and rapidly increases the capacity both of transport and of storage. If the layout of workplaces on both sides of the racks is designed well (replaceable workplaces always on one side, and the capacity of racks is satisfactory), the capacity can double. The difficulties with the breakdown of one stacker remain (at least one side of the centre must wait), but they are lowered.

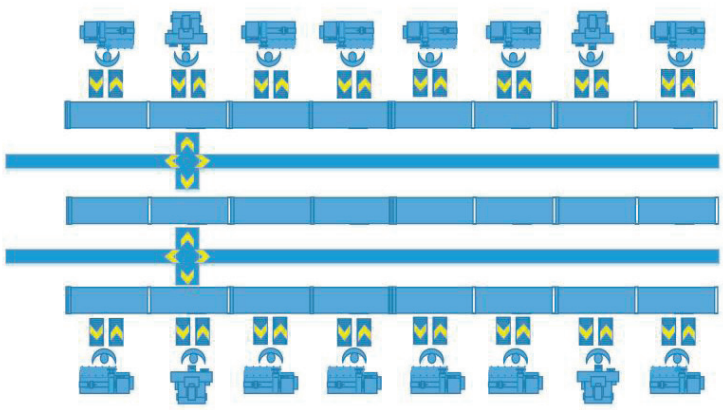

Fig. 2 Two stackers, racks on both sides, a middle rack, two aisles with one rail and workplaces on both sides of racks

Two stackers, a wider aisle, two rails close together and workplaces on both sides of racks

This solution for a Storage/Retrieval system (Fig. 3) was published in [3] with methods for how to coordinate these two stackers to prevent collisions. Stackers must pass by at different heights, cannot operate with forks on the same length positions and nearby. The shift out of the fork must reach both racks asymmetrically. The control algorithm is difficult, because the control systems of both stackers are not independent. The risk of collision by faulty sensors cannot be eliminated. On the other hand, if one stacker breaks down, the other could continue to work, while the first one waits in a parking position for service.

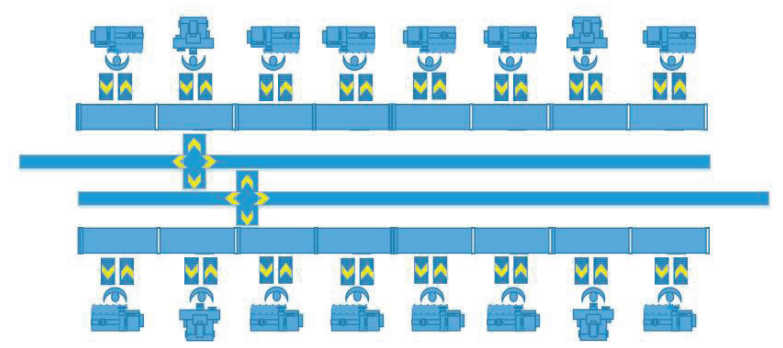

Fig. 3 Two stackers, a wider aisle, two rails close together and workplaces on both sides of racks

\section{Two stackers on one rail, one aisle, two racks and workplaces on both sides}

The solution is shown in Fig. 4. There is a minimum safety distance between stackers. A stacker in a parking position does not influence the other. If one stacker breaks down, the other one can continue to work, while the first one waits in a parking position for service. The control systems of both stackers are independent, there are only simple sensors detecting the minimum distance between stackers and changing the velocity in emergency to micro move.

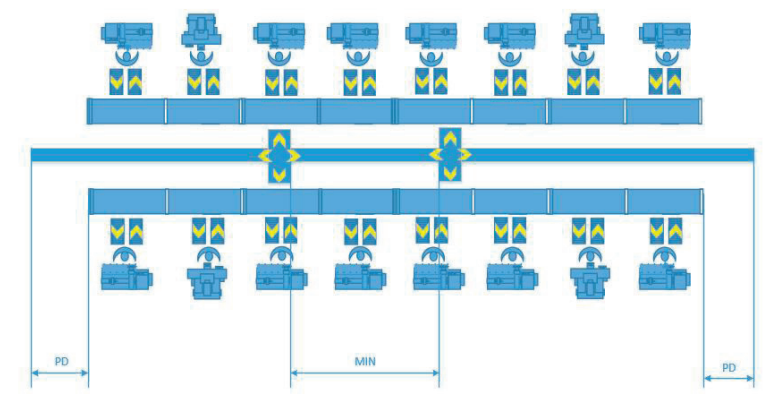

Fig. 4 Two stackers on one rail, one aisle, two racks and workplaces on both sides

\section{Optimization of the system with two stackers on one rail}

The most important question to be answered for optimization is the optimization criterion. Transport demands (start and end of each transport) are fixed. Losses are motions between the momentary position of the stacker and any start of transport. The criterion can then be the sum of the motion of the empty stackers or the total time of the execution of all given transport (makespan).

There are three levels of optimization:

1. Layout of the workplaces, so that e.g. workplaces from the beginning of the routing are on the left side, from the end 
are on the right side and workplaces more used at different positions in the routing (checking and measuring) are in the middle. Thus transport demands for long distances are reduced.

2. When searching for a free place in the racks, use of the "nearest place first" strategy (near can mean near to the input of a pallet in the transport system or near to the next workplace).

3. For a given set of demands, find an optimal order of demands for both stackers.

The first level should be implemented during the workshop layout proposal stage, and this proposal can be changed only with great difficulty.

The second level is simple to implement when generating a new transport demand after the signal "store in". Some problems could occur in the strategy "near to next workplace", when there are more possible (changeable) workplaces in the routing and they are placed far from each other.

The third level is the topic of this paper.

\section{Optimization possibilities}

As mentioned in [4], the optimal order of transport demands for one stacker is a NP problem. It is possible to find a solution by "brute force" only for a small number of demands (say 10). On the other hand, the "nearest demand first" algorithm is simple and the more demands it solves, the better are the results obtained.

This paper deals with "ad hoc" optimization strategies. Better methods for simulation optimization are discussed in [5]. Unfortunately, these methods are very sophisticated and time consuming and also need accurate production data.

The goal of the research was to prove that two stackers with a simple strategy could better solve both the increase of transport capacity and the reliability of the production system.

\section{Optimization experiments}

If both stackers are free, one of them is chosen randomly. If a stacker is free, a demand which does not have a planned collision

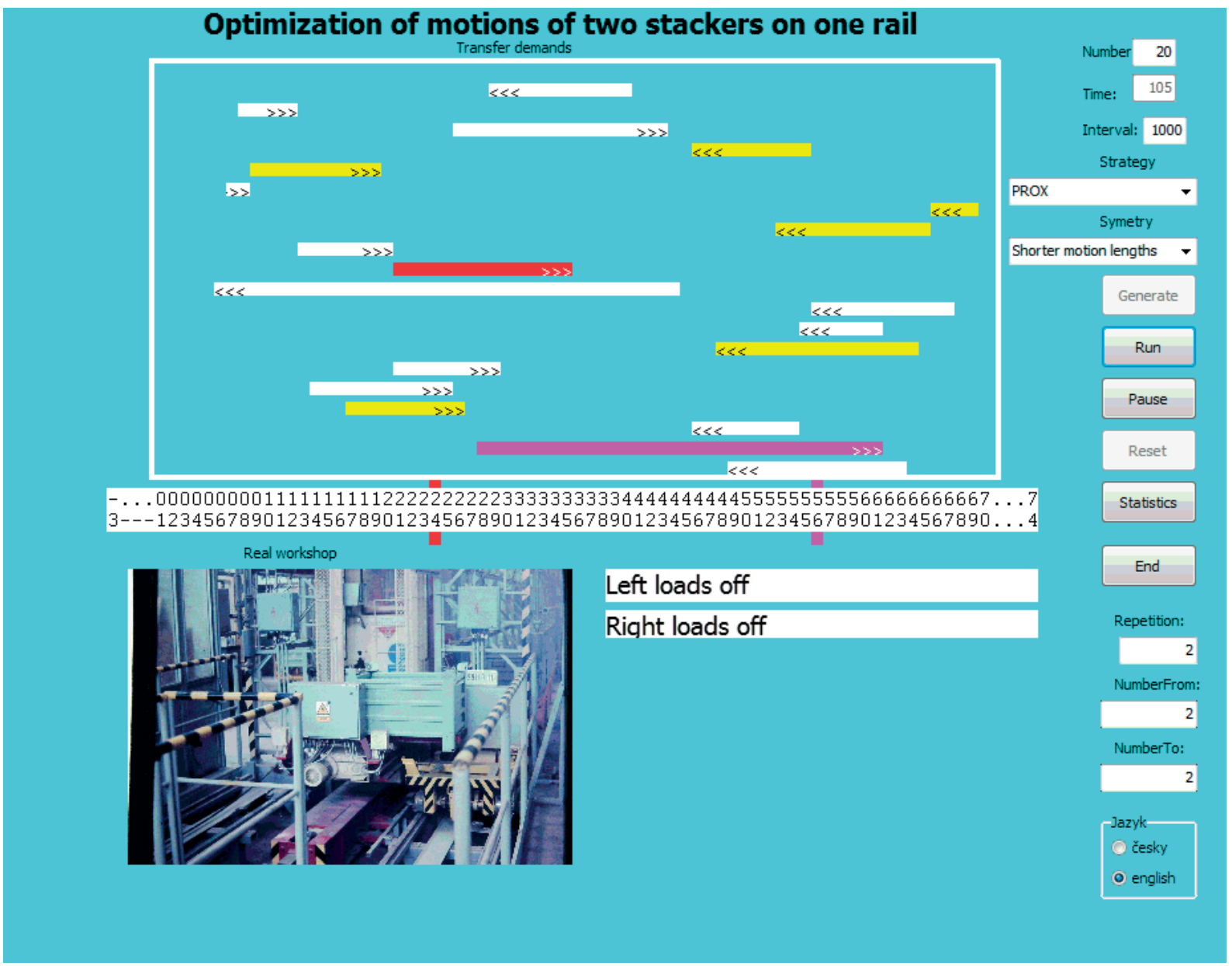

Fig. 5 Simulation program 
with the position of the other or with a movement of the other one is chosen.

Five ad hoc algorithms were tested ( 5 strategies):

1. FIFO: First demand fulfilling above mentioned condition is chosen.

2. PROX: The proximate demand fulfilling above mentioned condition is chosen.

3. BEST LEFT OR RIGHT: For the left stacker the most left ending demand is chosen, and for the right stacker the most right one.

4. COMB: Combination of NEAR and BEST LEFT OR RIGHT.

5. PROX and PAIRS: If both stackers are free, the pair with the sum of proximate starts is chosen.

The solution with only one stacker (the other one waits in the parking position in the state of breakdown) and the solution with both stackers were tested for each strategy.

One series of demands was tested with regular distribution of probability (every start and end position of any demand has the same probability, and neither optimization of workplace layout nor "nearest" strategy by finding a free place is used). The second series of demands regards layout and "near free place" strategy through shortening of lengths of $2 / 3$ of demands to $50 \%$.

The number of demands varies from 2 to 20 .

Experiments were very time demanding. Parallel computers have to be used. Each attempt is repeated 50 times. More repetitions did not generate more accurate results. Thus 5700 lines of results were obtained and transformed into graphs.

The program testing of the results from all the cases above was done with the animation of stacker motions and the possibility to accelerate or slow down the animation. The layout of the test program is shown in Fig. 5.

\section{Results and discussion}

Results of simulation are shown in Figs. 6 and 7.

Strategies "BEST LEFT OR RIGHT" and "COMB" are much better than FIFO, but not as good as the strategy "NEAR". "PROX and PAIRS" is also better than "PROX" but not much. For better clarity, they are not shown in Figs. 6 and 7 .

Figure 6 shows the regular distribution of probability for all generated starts and ends of transfers. The blue line (1-0) shows strategy "FIFO" with only one stacker, the red line (1-1) shows strategy "NEAR", the green line (2-1) shows strategy "FIFO" for two stackers and the violet line (2-2) shows strategy "NEAR" for two stackers

While one stacker with the FIFO strategy has fast, constant results (variations are only caused by the number of repetitions in the simulation), two stackers have better results (shorter manipulation times) for more demands. With this strategy, there is in practice no difference between two stackers with "FIFO" and one stacker with "NEAR".

Figure 7 shows transfers with regular distribution of probability for all generated starts and with shorter lengths of transport. The model on Fig. 7 should better cover the situation when the layout of the workplaces and the strategy of searching for free places in the racks are optimized. In this case the "NEAR" strategy is by far the best of all the strategies.

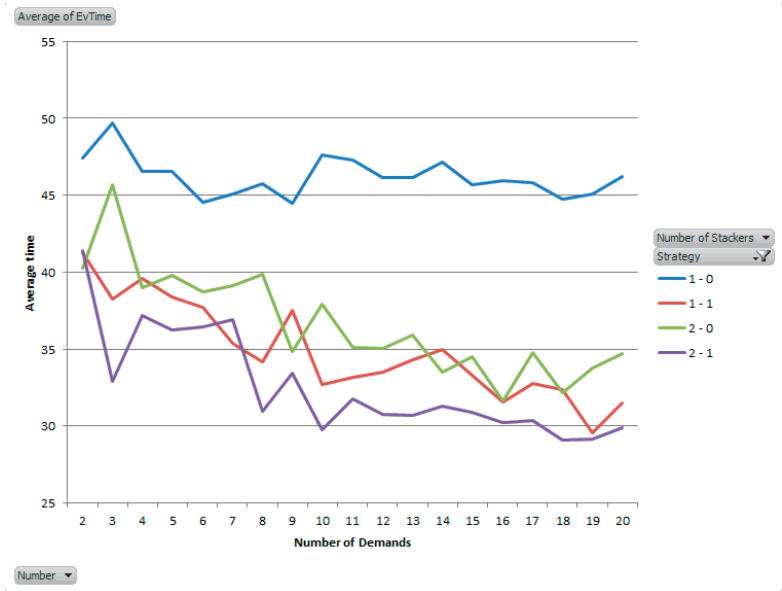

Fig. 6 Average time of one transport with regular distribution of starts and ends of any

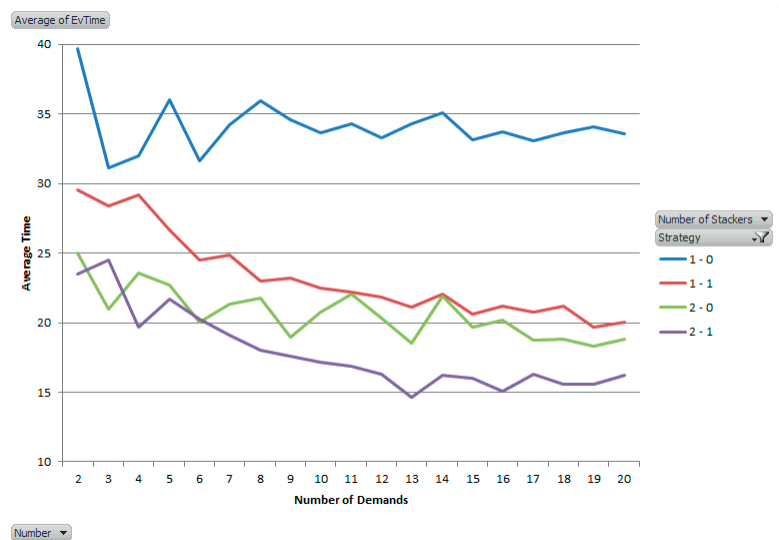

Fig. 7 Average time of one transport with regular distribution of starts of any transport and shortened lengths of transports

\section{Conclusion}

Though this paper describes only some ad hoc optimization algorithms, the results can be used in the design of new manufacturing systems for piece and low series production. Further research could focus on building optimal couples and using methods of simulation optimization [5], but sophisticated algorithms need accurate data and minimum disturbances in the real workshop, which cannot be guaranteed. 


\section{Acknowledgment}

The project presented in this article is supported by the project SGS-2015-065 (Development of parameters of a sustainable production system).
Results from the project OP VK n. CZ.1.07/2.3.00/09.0163 have been used.

\section{References}

[1] HALL, R. W.: The Architecture of Transportation Systems, Transpn. Res. C, vol. No. 3, pp. 129-142, Elsevier Science, 1995.

[2] LEE, S. G., DE SOUZA, R., ONG, E. K.: Simulation Modelling of a Narrow Aisle Automated Storage and Retrieval System (As/ Ds) Serviced by Rail/Guided Vehicles, Computers in Industry, 30, 1996, pp. 241-253, Elsevier, 1996.

[3] KUNG, Z., KOBAYASHI, Y., OTA, J.: Motion Planning of Two Stackers Cranes in a Large/Scale Automated Storage/Retrieval System, Proc. of the 2011 IEEE Intern. conference on Robotics and Biomechanics, December, 2011, Phuket, Thailand.

[4] KOPECEK, P., PINTE, M.: Optimization Heuristics for Supplies of Assembly Lines, Periodica Polytechnica Transportation Engineering, 2014, No. 1, pp. 49-52.

[5] RASKA, P., ULRYCH, Z.: Simulation Optimization in Manufacturing Systems, 'Annals of DAAAM for 2012 \& Proc. of the $23^{\text {rd }}$ Intern. DAAAM Symposium' (ed.: Katalinic B.), DAAAM International, Vienna, 221- 224 (2012). 